\title{
Divalent metal transporter, iron, and Parkinson's disease: A pathological relationship
}

\author{
Hyun-pil Lee ${ }^{1}$, Xiongwei Zhu ${ }^{1}$, Gang Liu ${ }^{2}$, Shu G Chen ${ }^{1}$, George Perry ${ }^{1,3}$, Mark A Smith ${ }^{1}$, Hyoung-gon Lee ${ }^{1}$ \\ ${ }^{1}$ Department of Pathology, Case Western Reserve University, Cleveland, Ohio 44106, USA; ${ }^{2}$ Department of Radiology, University of \\ Utah, Salt Lake City, UT, USA; ${ }^{3}$ UTSA Neuroscience Institute and Department of Biology, University of Texas at San Antonio, San \\ Antonio, Texas, USA
}

Cell Research (2010) 20:397-399. doi: 10.1038/cr.2010.39; published online 1 April 2010

Iron is an essential component of oxidative metabolism and a cofactor for a variety of enzymes. Because of its chemical properties as a transition metal, iron can serve both as an electron donor and acceptor and, as such, excess levels of free iron are toxic. Given this potential for toxicity, a number of proteins, including transferrin, transferrin receptor, and ferritin, tightly control iron transport, uptake, and storage in the central nervous system. Patients with Parkinson's disease (PD) show a dramatic increase in iron content in dopaminergic neurons of the substantia nigra, i.e., the neuronal population predominantly affected in PD patients. Dopamine is an important neurotransmitter regulating motor control such that loss of dopaminergic neurons results in the clinical symptomology typical of PD including resting tremor, postural instability, and poor coordination of general movements. Given the relationship of iron and dopaminergic neurons, it has been suggested that an imbalance of brain iron homeostasis contributes to the pathogenesis of PD. However, a major unresolved question concerns the molecular mechanism(s) involved in iron accumulation in the

\footnotetext{
Correspondence: Mark A Smith

Tel: 216-368-3671; Fax: 216-368-8964

E-mail:mark.smith@case.edu
}

brains of patients with PD. The recent study by Jiang and colleagues advances our understanding of this [1]. By using a PD animal model induced by 6-OHDA and a dopaminergic cell culture system, they provided direct evidence for a subtype of divalent metal transporter 1 (DMT1+IRE), containing iron response element (IRE) at its $3^{\prime}$ untranslated region, as a critical player in iron accumulation. Furthermore, they found that iron regulatory proteins (IRPs) regulate the induction of DMT1+IRE and that subsequent iron influx results in the functional loss of mitochondria and increase in oxidative stress.

As highlighted above, cellular iron homeostasis is tightly controlled by the coordinated expression of proteins involved in iron transport, uptake, export, and storage. Post-transcriptional control, mediated by the IRE/IRP system, has been suggested as a central mechanism to regulate these proteins. However, the IRE/IRP system of iron regulation is susceptible to oxidative stress [2], and the amount of IRP1 is, in turn, dependent on the cytosolic free iron concentration. In the absence of iron or under conditions of oxidative stress, IRP1 binds to the IREs of various iron proteins to regulate the translation of mRNA transcripts. Given the importance of iron in the pathogenesis of PD, it is clearly of importance to understand what leads to increased brain iron in PD.
In this regard, DMT1 might be a key regulator of brain iron accumulation in PD since both transferrin-dependent and -independent mechanisms for cellular transport of iron require DMT1 for the transport of iron. DMT1, also known as natural resistance associated macrophage protein 2 (Nramp2), is a widely expressed mammalian ferrous ion $\left(\mathrm{Fe}^{2+}\right)$ transporter [3]. Importantly, a recent study revealed that DMT1 is upregulated in the substantia nigra of both MPTP-induced PD models and PD patients [4], suggesting dysregulation of DMT1 might play a role in the accumulation of iron in PD. In the current paper, the authors found increase of DMT1+IRE and a positive correlation with iron accumulation in 6-OHDA-induced PD models which led them to further investigate the effect of DMT1+IRE expression on iron uptake in this PD model. Indeed, their data clearly show that the increased level of DMT1+IRE is associated with elevations in iron uptake and the production of reactive oxygen species and, consequently, oxidative stress. Pretreatment with the iron chelator desferrioxamine mesylate (DFO) abolished reactive oxygen species generation, supporting the role of DMT1 in iron accumulation in PD (Figure 1).

Interestingly, DMT1 is not only increased in $\mathrm{PD}$, but also colocalized with amyloid- $\beta$ in the senile plaques of 


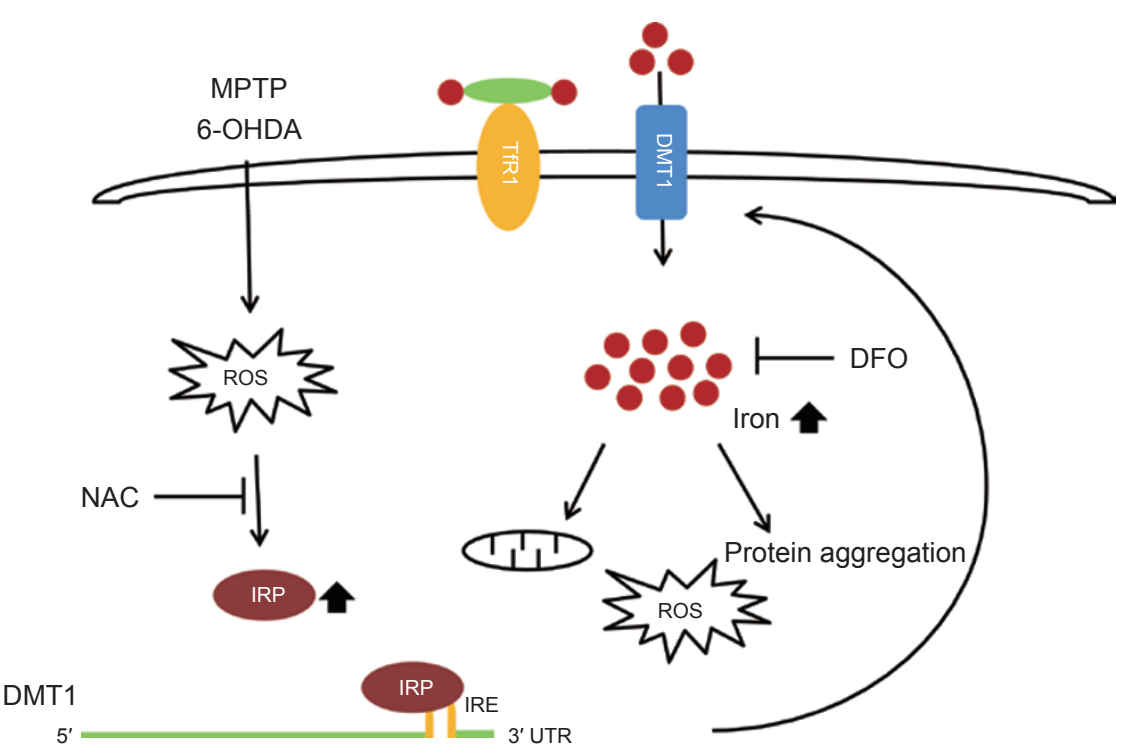

Figure 1 Oxidative stress induces DMT1 through the IRP/IRE system. DMT1 enhances iron influx which results in excess iron accumulation in neurons and subsequently induces mitochondrial dysfunction, reactive oxygen species, and protein aggregation. Therapeutic approaches using antioxidants such as NAC or iron chelators such as DFO, may protect neurons from iron-induced toxicity in PD. •: iron; TfR1: Transferrin receptor 1; DMT1: divalent metal transporter 1; IRP: iron regulatory protein; IRE: iron response element; NAC: $\mathrm{N}$-acetyl-cysteine; DFO: desferrioxamine; ROS: reactive oxygen species.

patients with Alzheimer's disease (AD) [5]. Iron homeostasis is also severely deranged in $\mathrm{AD}$, including increased redox active iron [6, 7], IRP [8], and other iron-regulator proteins [9]. Therefore, iron accumulation is a prominent feature in both types of neurodegenerative disease and DMT1 may play key pathogenic roles. Supporting this, iron accumulation has been implicated in the protein aggregations in both PD and $\mathrm{AD}$ such that removal of free iron with DFO can block $\alpha$-synuclein aggregation [10] and the metal chelators reverse amyloid aggregates in brain extracts from patients with $\mathrm{AD}[11]$ and also attenuate toxicity [12]. Given this, metal chelation may afford an opportunity to develop novel therapeutics as a means to delay the progression of these disorders. In this regard, we previously suggested that iron chelators conjugated with nanoparticles would enable chelators to cross the blood-brain barrier

\section{References}

1 Jiang H, Song N, Xu H, et al. Upregulation of divalent metal transporter 1 in 6-hydroxydopamine intoxication is IRE/IRP dependent. Cell Res 2010; 20:345-356.

2 Christova T, Templeton DM. Effect of hypoxia on the binding and subcellular distribution of iron regulatory proteins. Mol Cell Biochem 2007; 301:21-32.

3 Gunshin H, Mackenzie B, Berger UV, et al. Cloning and characterization of a mammalian proton-coupled metal-ion transporter. Nature 1997; 388:482-488.

4 Salazar J, Mena N, Hunot S, et al. Divalent metal transporter 1 (DMT1) contributes to neurodegeneration in animal models of Parkinson's disease. Proc Natl Acad Sci USA 2008; 105:1857818583.

5 Zheng W, Xin N, Chi ZH, et al. Divalent metal transporter 1 is involved in amyloid precursor protein processing and Abeta generation. FASEB J 2009; 23:4207-4217.

6 Smith MA, Harris PL, Sayre LM, Perry G. Iron accumulation in Alzheimer disease is a source of redox-generated free radicals. Proc Natl Acad Sci USA 1997; 94:9866-9868.

7 Sayre LM, Perry G, Harris PL, et al. In situ oxidative catalysis by neurofibrillary tangles and senile plaques in Alzheimer's disease: a central role for bound transition metals. $J$ Neurochem 2000; 74:270-279.

8 Smith MA, Wehr K, Harris PL, et al. Abnormal localization of iron regulatory protein in Alzheimer's disease. Brain Res 1998; 788:232-236.

9 Connor JR, Menzies SL, St Martin SM, Mufson EJ. A histochemical study of iron, transferrin, and ferritin in Alzheimer's diseased brains. J Neurosci Res 1992; 31:75-83.

10 Sangchot P, Sharma S, Chetsawang B, et al. Deferoxamine attenuates ironinduced oxidative stress and prevents mitochondrial aggregation and alphasynuclein translocation in SK-N-SH cells in culture. Dev Neurosci 2002; 24:143-153.

11 Cherny RA, Legg JT, McLean CA, et al. Aqueous dissolution of Alzheimer's disease Abeta amyloid deposits by biometal depletion. J Biol Chem 1999; 274:23223-23228.

12 Rottkamp CA, Raina AK, Zhu X, et al. 
Redox-active iron mediates amyloidbeta toxicity. Free Radic Biol Med 2001; 30:447-450.
13 Liu G, Men P, Perry G, Smith MA. Nanoparticle and iron chelators as a potential novel Alzheimer therapy. Methods Mol Biol 2010; 610:123-144. 